\title{
Signatures of Intergalactic Dust from the First Supernovae
}

\section{Citation}

Loeb, Abraham, and Zoltan Haiman. 1997. "Signatures of Intergalactic Dust from the First Supernovae.” The Astrophysical Journal 490 (2): 571-76. https://doi.org/10.1086/304919.

\section{Permanent link}

http://nrs.harvard.edu/urn-3:HUL.InstRepos:41393180

\section{Terms of Use}

This article was downloaded from Harvard University's DASH repository, and is made available under the terms and conditions applicable to Other Posted Material, as set forth at http:// nrs.harvard.edu/urn-3:HUL.InstRepos:dash.current.terms-of-use\#LAA

\section{Share Your Story}

The Harvard community has made this article openly available.

Please share how this access benefits you. Submit a story.

\section{Accessibility}


The Astrophysical Journal, 490:571-576, 1997 December 1

(C) 1997. The American Astronomical Society. All rights reserved. Printed in U.S.A.

\title{
SIGNATURES OF INTERGALACTIC DUST FROM THE FIRST SUPERNOVAE
}

\author{
Abraham Loeb AND Zoltán Haiman \\ Astronomy Department, Harvard University, 60 Garden Street, Cambridge, MA 02138 \\ Received 1997 April 14; accepted 1997 July 14
}

\begin{abstract}
We quantify the consequences of intergalactic dust produced by the first Type II supernovae in the universe. The fraction of gas converted into stars is calibrated based on the observed $\mathrm{C} / \mathrm{H}$ ratio in the intergalactic medium at $z=3$, assuming a Scalo mass function for the stars. The associated dust absorbs starlight energy and emits it at longer wavelengths. For a uniform mix of metals and dust with the intergalactic gas, we find that the dust distorts the microwave background spectrum by a $y$-parameter in the range $(0.06-6) \times 10^{-5}\left(M_{\mathrm{SN}} / 0.3 M_{\odot}\right)$, where $M_{\mathrm{SN}}$ is the average mass of dust produced per supernova. The opacity of intergalactic dust to infrared sources at redshifts of $z \gtrsim 10$ is significant, $\tau_{\text {dust }}=(0.1-1)$ $\times\left(M_{\mathrm{SN}} / 0.3 M_{\odot}\right)$, and could be detected with the Next Generation Space Telescope. Although dust suppresses the Ly $\alpha$ emission from early sources, the redshifts of star clusters at $z=10-35$ can be easily inferred from the Lyman limit break in their infrared spectrum between 1 and $3.5 \mu \mathrm{m}$.
\end{abstract}

Subject headings: cosmology: theory — dust, extinction — galaxies: ISM — infrared: galaxies supernovae: general

\section{INTRODUCTION}

Recent spectroscopic observations of the $\operatorname{Ly} \alpha$ forest at $z \gtrsim 3$ show evidence for a metallicity of $\sim 1 \% Z_{\odot}$ in absorbers with $\mathrm{H}$ I column densities as low as $10^{15} \mathrm{~cm}^{-2}$ (Cowie 1996; Songaila \& Cowie 1996; Tytler et al. 1995). Numerical simulations identify such absorbers with mildly overdense regions in the intergalactic medium (IGM), out of which nonlinear objects such as galaxies condense (Hellsten et al. 1997 and references therein). Indeed, damped Ly $\alpha$ absorbers, which are thought to be the progenitors of present-day galaxies, show similar metalicities during the early phase of their formation at $3.5 \lesssim z \lesssim 4.5$ ( Lu et al. 1996). The universality of this mean metal abundance in absorbers that span a range of 6 orders of magnitude in $\mathrm{H}_{\mathrm{I}}$ column density indicates that an early phase of metal enrichment occurred throughout the universe at $z \gtrsim$ 5 , before galaxies were assembled.

The formation of an early population of star clusters at redshifts $\sim 10-30$ is a natural consequence of hierarchical structure formation in cold dark matter (CDM) cosmologies (see, e.g., Haiman \& Loeb 1997, hereafter HL97; Ostriker \& Gnedin 1996 and references therein). The first star clusters that form in high abundance have baryonic masses of $\sim 10^{8} M_{\odot}$ and virial temperatures of $\sim 10^{4} \mathrm{~K}$, which allow their gas to cool via atomic transitions. Less massive objects with lower virial temperatures are unable to cool and fragment into stars because of the rapid photodissociation of their molecular hydrogen (Haiman, Rees, \& Loeb 1996). Since the potential wells of the first clusters are relatively shallow $\left(\sim 10 \mathrm{~km} \mathrm{~s}^{-1}\right)$, supernova-driven winds might have expelled the metal-rich gas out of these systems and mixed it with the IGM. Incomplete mixing could have led to the observed order-of-magnitude scatter in the $\mathrm{C} / \mathrm{H}$ ratio along lines of sight to different quasars (Rauch, Haehnelt, \& Steinmetz 1997; Hellsten et al. 1997). It is an interesting coincidence that the supernova energy output associated with a metal enrichment of the IGM by $\sim 1 \%$ $Z_{\odot}$ corresponds to an energy release of $\sim 10 \mathrm{eV}$ per hydrogen atom in the IGM, which is just above the binding energy of these early star clusters. Supernova feedback in these objects could have therefore determined the average metallicity observed in the Ly $\alpha$ forest. Direct observations of these supernovae might be feasible in the future (MiraldaEscudé \& Rees 1997).

The measured $\mathrm{C} / \mathrm{H}$ ratio can be used to calibrate the net fraction of gas that is converted into stars by a redshift of $z \approx 3$. In HL97, we have used this fraction and an extension of the Press-Schechter formalism to calculate the early star formation history in a variety of CDM cosmologies. For a large range of models we have found that the universe is reionized by a redshift of $z=10-20$ and that the resulting optical depth to electron scattering, $\sim 0.1$, will be detectable with future microwave anisotropy experiments. In addition, deep imaging with future infrared telescopes, such as the Space Infrared Telescope Facility (SIRTF) or the Next Generation Space Telescope (NGST), would be able to detect pregalactic star clusters. In particular, NGST should find $\gtrsim 10^{3}$ star clusters per square arcminute at $z>10$, given its planned detection threshold of $1 \mathrm{nJy}$ in the wavelength range of 1-3.5 $\mu \mathrm{m}$ (Mather \& Stockman 1996).

The early epoch of star formation and metal enrichment is inevitably accompanied by the formation of dust in supernova shells. This dust has two important observational signatures. First, the absorption of starlight energy and its re-emission at long wavelengths distorts the spectrum of the cosmic microwave background (CMB) radiation (Wright et al. 1994; Bond, Carr \& Hogan 1991; Adams et al. 1989; Wright 1981). Second, the opacity of the intergalactic medium to infrared sources at redshifts of $z \gtrsim 10$ could be significant. For these redshifts, infrared in the observer frame corresponds to UV in the source frame-a spectral regime in which dust absorption peaks. ${ }^{1}$ Dust obscuration must therefore be considered when predicting the performance of future infrared telescopes such as NGST.

In this paper, we quantify the magnitude of the above signatures of intergalactic dust. In $\S 2$ we describe our method of calculation, and in $\S 3$ we compute the distortion of the microwave background spectrum and compare it to

\footnotetext{
${ }^{1}$ The effect of intergalactic dust at much lower redshifts was discussed in the context of the reddening (Cheney \& Rowan-Robinson 1981; Wright 1981, 1990) and the number count (Ostriker \& Heisler 1984; Ostriker, Vogeley, \& York 1990) of quasars.
} 
the current $C O B E$ limit. In $\S 4$ we evaluate the opacity of the intergalactic medium to infrared sources. Finally, $\S 5$ summarizes the implications of this work for future microwave background and infrared observations. Throughout the paper, we adopt a Hubble constant of $H_{0}=50 \mathrm{~km} \mathrm{~s}^{-1}$ $\mathrm{Mpc}^{-1}$ and a cosmological density parameter, $\Omega=1$.

\section{METHOD OF CALCULATION}

Our first goal is to calculate the distortion of the CMB spectrum due to the production of radiation by stars and its processing through dust. The CMB spectrum is initially a pure blackbody with a temperature of $T_{\mathrm{CMB}}=2.728(1+z)$ K (Fixsen et al. 1996). We assume that stars are born with the Galactic initial mass function (IMF) and use the sixsegment power-law parametrization between 0.1 and 125 $M_{\odot}$ given by Scalo (1986) (see also Fig. 9 of HL97). We compute a composite time-dependent spectrum using standard spectral atlases (Kurucz 1993) and evolutionary tracks (Schaller et al. 1992). Our stellar modeling is described in detail in HL97. In computing the amount of dust produced by the stars, we assume that each Type II supernova yields $0.3 M_{\odot}$ of dust, which gets uniformly distributed in the intergalactic medium (see discussion in $\S 3$ ), and that the dust absorption follows the wavelength-dependent opacity of Galactic dust.

To describe the effect of dust on the CMB, we follow Wright (1981) and define the comoving number density of photons with a comoving frequency, $v$, as

$$
N_{v}(z) \equiv \frac{4 \pi}{h c(1+z)^{3}} J_{v(1+z)}(z),
$$

where $J_{v}(z)$ is the specific intensity of the background radiation field (microwave background + starlight + dust emission) in ergs $\mathrm{cm}^{-2} \mathrm{~s}^{-1} \mathrm{~Hz}^{-1} \mathrm{sr}^{-1}$. The evolution of $N_{v}(z)$ is determined by the radiative transfer equation,

$$
-\frac{d N_{v}(z)}{d z}=\frac{c d t}{d z}\left[j_{v}(z)-\alpha_{v(1+z)}(z) N_{v}(z)\right],
$$

where, in an $\Omega=1$ universe, $(c d t / d z)=\left(c / H_{0}\right)(1+z)^{-5 / 2}$. Here, $j_{v}(z)$ is the comoving emission coefficient due to stars and dust,

$$
\begin{aligned}
j_{v}(z)= & \frac{4 \pi}{h c(1+z)^{3}} j_{v(1+z)}^{*}(z)+\frac{8 \pi}{c^{3}} \\
& \times\left\{\frac{v^{3}}{\exp \left[h v(1+z) / k_{\mathrm{B}} T_{\text {dust }}\right]-1}\right\} \alpha_{v(1+z)}(z),
\end{aligned}
$$

and $\alpha_{v}(z)$ is the dust absorption coefficient,

$$
\alpha_{v}(z)=\rho_{\text {dust }}(z) \kappa_{v},
$$

for a mass density, $\rho_{\text {dust }}$, in dust. We assume that the dust is in thermal equilibrium with the total radiation field and equate the power it absorbs in the UV to the power it emits in the infrared. The dust temperature, $T_{\text {dust }}$, as a function of redshift is then derived from the implicit equation,

$$
\begin{aligned}
& \int_{0}^{\infty} d v \kappa_{v(1+z)} \\
& \quad \times\left\{N_{v}(z)-\frac{8 \pi}{c^{3}} \frac{v^{3}}{\exp \left[h v(1+z) / k_{\mathrm{B}} T_{\text {dust }}\right]-1}\right\}=0,
\end{aligned}
$$

where $N_{v}(z)$ is the solution of the radiative transfer equation [eq. (2)]. We adopt the dust opacity, $\kappa_{v}$, based on the mean Galactic extinction law, $A(\lambda) / A(J)$, of Mathis (1990),

$$
\begin{aligned}
\kappa_{v}=8.3 \times 10^{3}\left[\frac{A(\lambda)}{A(J)}\right] \\
\times\left\{1-\frac{0.084}{0.12+[\lambda(\mu \mathrm{m})-0.4]^{2}}\right\} \mathrm{cm}^{2} \mathrm{~g}^{-1},
\end{aligned}
$$

where $\lambda=c / v$ is the wavelength; the numerical coefficient converts the extinction per hydrogen atom, with the assumed gas-to-dust ratio of 100, to extinction per gram of dust. The last term is the albedo term given by Wright (1981) for converting the extinction (absorption plus scattering) to pure absorption. We find that our results change by less than $5 \%$ if we use the sum of the theoretical graphite + silicate grain cross sections derived by Draine \& Lee (1984).

Given a specific star formation history, stellar spectra, and dust yields, one can integrate numerically equations (2)-(5) and get the dust temperature and the net spectrum of the radiation background at $z=0$. As in HL97, we express the star formation history in terms of the mass fraction of all baryons that are assembled into collapsed objects, $F_{\text {coll }}(z)$, and the fraction of these collapsed baryons that get incorporated into stars, $f_{\text {star }}$. The density parameter of stars is then $\Omega_{\text {star }}(z)=f_{\text {star }} F_{\text {coll }}(z) \Omega_{\mathrm{b}}$, where $\Omega_{b}$ is the total baryonic density parameter $(=0.05$ in our standard model). The collapsed fraction, $F_{\text {coll }}(z)$, is calculated in a standard CDM cosmology based on an extension of the Press-Schechter formalism that takes into account pressure and photodissociation of $\mathrm{H}_{2}$ (HL97; Haiman, Thoul, \& Loeb 1996). To study the effects of high-redshift stars alone, we do not allow stars to form at $z<3$, i.e., we set $F_{\text {coll }}(z<3) \equiv$ $F_{\text {coll }}(z=3)$. The contributions to the UV flux from quasars and stars at $z<3$ that are ignored here could slightly increase the spectral distortion we derive.

The star formation efficiency is found to be $f_{\text {star }} \sim 12 \%$ from the requirement that the $\mathrm{C} / \mathrm{H}$ ratio be $1 \%$ of the solar value at $z=3$. Since the collapsed fraction at $z=3$ is $\sim 50 \%$, the corresponding fraction of all baryons in stars is $\sim 6 \%$. A factor of $\sim 2.3$ is included in this number owing to the average time required to produce carbon inside the stars, which we assume is the main-sequence time. Under this assumption, only $\sim 43 \%$ of the total stellar carbon is produced by $z=3$. As emphasized in HL97, carbon is mostly produced by intermediate mass $\left(3-6 M_{\odot}\right)$ stars, whereas dust is heated primarily by the high-mass stars. Our modeling is therefore sensitive to the adopted slope of the IMF; metallicity measurements at high redshifts for elements other than carbon would be useful in constraining the IMF at high redshifts.

The stellar emission coefficient, $j_{v}^{*}(z)$, is found at each redshift, $z$, as a sum of the instantaneous emission from all the stars present at $z$,

$$
j_{v}^{*}(z)=f_{\mathrm{star}} \Omega_{b} \rho_{c 0}(1+z)^{3} \int_{z}^{\infty} d z^{\prime} \frac{d F_{\text {coll }}}{d z^{\prime}} \epsilon^{*}\left[v\left(1+z^{\prime}\right), t_{z, z^{\prime}}\right],
$$

where $\rho_{c 0}$ is the current critical density of the universe, $t_{z, z^{\prime}}$ is the time interval between the redshift $z^{\prime}$ at which a star was born and the redshift $z$, and $\epsilon^{*}(v, t)$ is the composite physical emissivity (in $\operatorname{ergs}^{-1} \mathrm{~Hz}^{-1} M_{\odot}^{-1}$ ) of a cluster of 
stars with the Scalo IMF at time $t$ after its initial starburst (see HL97 for details). We have assumed that all stellar photons escape their parent clouds and propagate into the intergalactic medium. In reality, a fraction of the ionizing photons will get converted into line photons with energies $E<13.6 \mathrm{eV}$ by case B recombinations inside the clouds, changing the emerging stellar spectrum. Since only a fraction of about one-third of the stellar energy output is emitted above $13.6 \mathrm{eV}$ and the dust absorption cross sections at 13.6 and $10.2 \mathrm{eV}$ differ only by $\sim 30 \%$, this will only have a minor $(\sim 10 \%)$ effect on the total amount of energy absorbed by dust. Free-free emission by the ionized gas in the cluster can be neglected relative to the stellar emission. The mass density of dust at each redshift is given by

$$
\rho_{\text {dust }}(z)=f_{\text {dust }} f_{\text {star }} \Omega_{\mathrm{b}} \rho_{\mathrm{co}}(1+z)^{3} \int_{z}^{\infty} d z^{\prime} \frac{d F_{\text {coll }}}{d z^{\prime}} f_{\text {dep }}\left(t_{z, z^{\prime}}\right),
$$

where $f_{\text {dust }}$ is the mass fraction of stars that gets converted into dust and $0 \leq f_{\text {dep }}(t) \leq 1$ is the fraction of the total mass in dust that is deposited after a time $t$ following the starburst (i.e., the mass fraction of the $\geq 8 M_{\odot}$ stars that have completed their main-sequence lifetime). Assuming that each supernova in a Scalo mass function produces $0.3 M_{\odot}$ of dust leads to $f_{\text {dust }}=0.001$. The uncertainties in this value will be discussed in the next section.

\section{DISTORTION OF THE MICROWAVE BACKGROUND SPECTRUM RESULTING FROM DUST EMISSION}

To parameterize the deviation of the spectrum at $z=0$ from a $T_{\mathrm{CMB}}=2.728 \mathrm{~K}$ blackbody, we compute the Compton $y$-parameter, $y_{\mathrm{C}}$, defined as

$$
y_{\mathrm{C}} \equiv \frac{1}{4}\left(\frac{\int d v N_{v}}{\int d v N_{0, v}}-1\right),
$$

where the integrals are evaluated over the FIRAS frequency range of 60-600 GHz (Fixsen et al. 1996), and

$$
N_{0, v}=\frac{8 \pi}{c^{3}} \frac{v^{3}}{\exp \left(h v / k_{\mathrm{B}} T_{\mathrm{CMB}}\right)-1} .
$$

The $y$-parameter we derive is subject to a number of uncertainties associated with the formation of dust. Each of these uncertainties can be parameterized by a separate factor that multiplies our result for $y_{\mathrm{C}}$. The first uncertainty involves the average fraction of the total stellar mass that gets converted into dust, $f_{\text {dust }}$. The value, $f_{\text {dust }}=0.001$, adopted in $\S 2$ is equivalent to assuming that each star with mass above $8 M_{\odot}$ explodes as a supernova and produces 0.3 $M_{\odot}$ of dust. This mass is consistent with the values inferred from observations of the depletion of the Si I line in SN 1987A (Lucy et al. 1991). Theoretical estimates with $100 \%$ condensation efficiency predict values as high as $1 M_{\odot}$ of dust per supernova (Dwek 1988; Weaver \& Woosley 1980). If the dust yield of supernovae is supplemented by a significant amount of dust produced in stars, the value of $f_{\text {dust }}$ could increase even further. On the other hand, a substantial fraction of the dust may be destroyed by sputtering inside the supernova cavity or by shock waves from other supernovae (Dwek 1997); the $y$-parameter scales linearly with the survival fraction of dust, $f_{\text {surv }}$. The $y$-parameter would also be affected by a spatial correlation between the distributions of dust and stellar sources. If dust is correlated with star-forming regions, the radiation flux probed by the dust could be enhanced by a factor, $f_{\text {flux }}$, relative to the average flux in equation (7). Note that $f_{\text {flux }}>1$ as long as the dust surrounding the star-forming region is optically thin; a full treatment of the three-dimensional geometry of optically thick clumps is beyond the scope of the present paper. For small values of $f_{\text {flux }}, y_{\mathrm{C}}$ scales linearly with $f_{\text {flux }}$. A related uncertainty involves the mixing efficiency of carbon with the intergalactic gas. The star formation efficiency, $f_{\text {star }}$, that is required in order to get the observed metallicity of the $\mathrm{Ly} \alpha$ forest is inversely proportional to this mixing efficiency. From equations (2), (3), and (7), we get $y_{\mathrm{C}} \propto f_{\text {star }}^{2}$, since the radiation emitted by dust is proportional to its opacity and the stellar radiation background-both of which are independently proportional to $f_{\text {star. }}$. Finally, an additional uncertainty is due to the processing of the emergent stellar spectrum by neutral hydrogen, as discussed above. Depending on the amount and three-dimensional geometry of hydrogen present inside the star-forming regions, this additional processing of starlight may reduce the amount of energy absorbed by dust by a factor of $f_{\text {spec }}$. All of these uncertainties can be combined through the definition of an overall efficiency parameter, $\eta_{y}$ :

$$
\eta_{\mathrm{y}} \equiv f_{\text {surv }} f_{\text {flux }} f_{\text {spec }}\left(\frac{f_{\text {dust }}}{0.001}\right)\left(\frac{f_{\text {star }}}{0.13}\right)^{2},
$$

so that $y_{\mathrm{C}} \propto \eta_{y}$, and $\eta_{y} \equiv 1$ in our standard model.

In addition to the uncertainties about the dust and the star formation efficiency, we note that if an early generation of low-luminosity quasars of the type discussed by Eisenstein \& Loeb (1995) exists, these quasars could have produced UV radiation exceeding the stellar output. The UV light from these quasars would be reprocessed by dust in much the same way as the stellar radiation is and could substantially increase the spectral distortion derived from the stars alone. The possible effects of early quasars are ignored here and will be quantified in a future paper.

Finally, there are two uncertainties related to the absorption cross section of dust: (1) the mean Galactic extinction law of Mathis (1990) is uncertain above $30 \mu \mathrm{m}$, and (2) theoretical modeling of the extinction law (Draine \& Lee 1984) indicates that graphite and silicate dust grains contribute roughly the same amount to the Galactic extinction, with different individual cross sections. To address these issues, we repeated our calculations (1) with the Mathis extinction law modified to $A(\lambda>30 \mu \mathrm{m}) \sim 1 / \lambda^{2}$ and (2) including graphite and silicate dust in the respective proportions $47 \%$ and $53 \%$ as found by Draine \& Lee (1984) and allowing the two components independently to reach their equilibrium temperatures. In the first case, we find that changing the cross section reduces the $y$-parameter by less than $2 \%$, since the cross section is changed only in the wavelength range that is $\sim 3$ orders of magnitude away from the peak. In the second case, we find equilibrium temperatures of $T=6.1$ and $4.3 \mathrm{~K}$ at $z=0$ for graphite and silicate, respectively, which differ from the temperature of $T=5.6 \mathrm{~K}$ when a single temperature is assumed. However, despite this difference in temperatures, the $y$-parameter is reduced by less than $3 \%$ in the $C O B E$ regime of the spectrum. Thus, both of the above uncertainties have little effect on our results.

Figure 1 shows the full spectrum of the background radiation for our standard model, whose parameters are sum- 


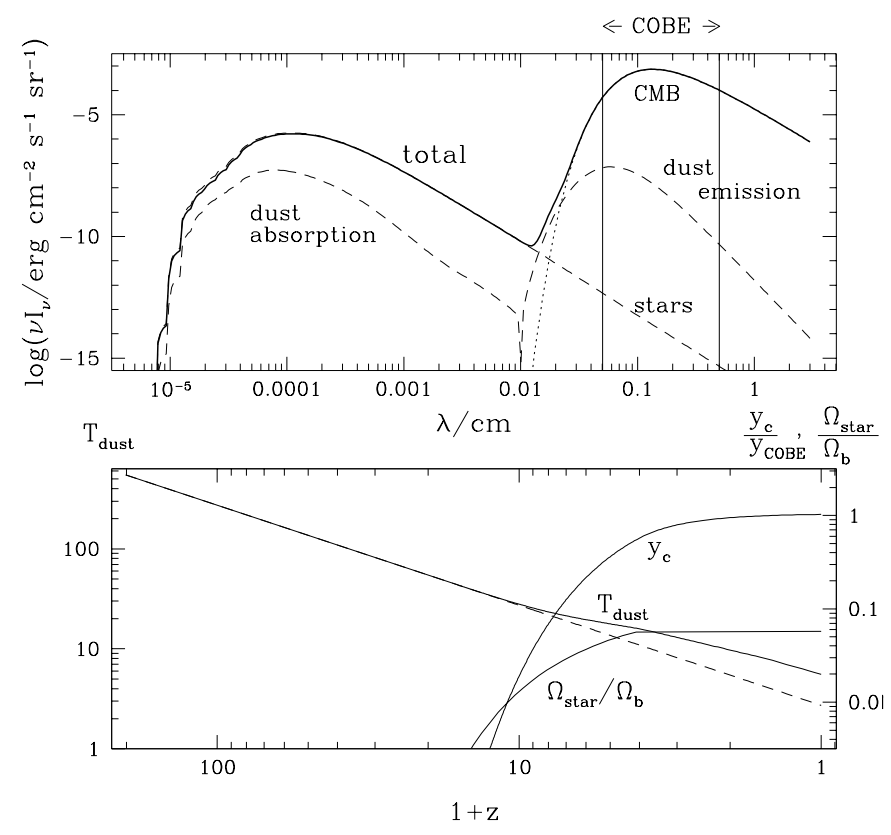

Fig. 1.-Top panel: Full spectrum at $z=0$ in our standard model for the star formation history. The solid line shows the total spectrum, and the dotted and dashed lines show the separate contributions of the original $\mathrm{CMB}$, the stars, and the dust. The net contribution of dust results in absorption at $\lambda \lesssim 0.015 \mathrm{~cm}$ and emission at longer wavelengths. Bottom panel: Redshift evolution of the fraction of baryons in stars, the dust temperature, and the $y$-parameter. Note that $f_{\text {star }} \equiv F_{\text {coll }}(z)^{-1} \Omega_{\text {star }} / \Omega_{b}$. The dashed curve shows the history of the CMB temperature.

marized in Table 1. The solid line depicts the total spectrum, which is a sum over the contributions from the original CMB, the stars, and the dust; the individual contributions of the CMB, the stars, and the dust are shown separately by the dotted and dashed lines. The net contribution from dust is negative (absorption) for $\lambda \lesssim 0.015 \mathrm{~cm}$. The dust emission at longer wavelengths peaks around $\lambda \approx 0.1 \mathrm{~cm}$, within the $C O B E$ range. The $y$-parameter derived from this spectrum is $1.54 \times 10^{-5}$, which is comparable to the upper limit of $1.5 \times 10^{-5}$ established by $C O B E$ (Fixsen et al. 1996).

For reference, the bottom panel of Figure 1 shows the star formation history, i.e., the fraction of baryons confined in stars as a function of redshift, for our standard model. The same panel also shows the histories of the dust temperature and the $y$-parameter. The dust temperature starts to deviate noticeably from the CMB temperature (dashed line) around $z \approx 10$, when the fraction of baryons in stars $\Omega_{\text {star }} / \Omega_{b}=f_{\text {star }} F_{\text {coll }} \sim 2 \%$. Note that we ignore stars and dust that form at redshifts of $z \leq 3$, so that the dust emission from lower redshifts is from preexisting dust that was heated prior to $z=3$. Our calculation is inadequate at low redshifts, since it ignores the enhanced production of metals and dust inside galaxies and the contribution of quasars to the background radiation. The spectral distortion we obtain should therefore be regarded as a lower bound; inclusion of the contribution from lower redshifts could only increase its magnitude.

Table 1 shows the range of values obtained for the $y$ parameter as one deviates from the input parameters assumed in our standard model. In particular, if the normalization of the standard CDM power spectrum, $\sigma_{8 h^{-1}}$, is increased, then star formation starts earlier, which tends to increase the optical depth because of the higher density. On the other hand, the star formation efficiency in this case is reduced by $30 \%$ relative to our standard model because of the longer time available for the stars to produce carbon. The net result is an increase of the optical depth by only $5 \%$ but a decrease of the corresponding $y$-parameter by $\sim 35 \%$, since star formation is now spread over a wider range of redshifts, and the average time delay between the production of radiation and dust is increased.

Tilting the power spectrum to have a lower power-law index, $n$, has the opposite effect; stars are produced later and over a narrower range of redshifts, and the star formation efficiency has to be increased by $\sim 70 \%$ because of the increased importance of the delay in the carbon production. In this case, the optical depth is increased by $20 \%$, but the $y$-parameter increases by more than a factor of 2 . Since we normalize the star formation efficiency by fixing the total carbon yield of the stars, a change to the slope of the IMF has an effect on the net production of dust. Adding a constant, $-0.7 \leq \beta \leq 1$, to the power-law index of the IMF changes the $y$-parameter in the range $0.62 \leq\left(y_{\mathrm{C}} / 10^{-5} \eta_{y}\right) \leq$ 1.98 .

When the photodissociation of $\mathrm{H}_{2}$ is ignored (e.g., owing to the renewed formation of $\mathrm{H}_{2}$ on dust grains), the threshold for fragmentation and star formation in virialized objects is lowered from virial temperatures of $T_{\mathrm{vir}} \gtrsim 10^{4} \mathrm{~K}$ (where atomic line cooling is effective) to $T_{\mathrm{vir}} \gtrsim 10^{2} \mathrm{~K}$ (where $\mathrm{H}_{2}$ cooling operates). The effect is similar to increasing $\sigma_{8 h^{-1}}$, i.e., star formation starts earlier, but $f_{\text {star }}$ is decreased by $\sim 40 \%$. The net results are a slight increase in the optical depth but a decrease in the $y$-parameter to $y_{\mathrm{C}}=$ 0.77. The input parameter that affects $y_{\mathrm{C}}$ the most is the baryon density, $y_{\mathrm{C}} \propto \Omega^{2}{ }_{b}$; high values of $\Omega_{b}$ (such as $\Omega_{b} \gtrsim$ 0.05 , if all other parameters are held fixed) violate the existing $C O B E$ constraint.

TABLE 1

Parameter Values in Our Standard Model and Its Variants

\begin{tabular}{ccccc}
\hline \hline Parameter & Standard & Range Considered & Compton $y_{\mathrm{C}} / 10^{-5} \eta_{y}$ & $\begin{array}{c}\text { Optical Depth } \\
\tau_{\text {dust }} / \eta_{\tau}\end{array}$ \\
\hline$\sigma_{8 h-1} \ldots \ldots \ldots \ldots \ldots$ & 0.67 & $0.67-1.0$ & $1.54-0.96$ & $0.42-0.44$ \\
$n \ldots \ldots \ldots \ldots \ldots$. & 1.0 & $0.8-1.0$ & $3.36-1.54$ & $0.50-0.42$ \\
IMF tilt $(\beta) \ldots \ldots$. & 0 & $-0.7-1.0$ & $0.62-1.98$ & $0.17-0.55$ \\
$\mathrm{H}_{2}$ feedback $\ldots \ldots$. & Yes & Yes $/$ no & $1.54-0.77$ & $0.40-0.45$ \\
$\Omega_{b} \ldots \ldots \ldots \ldots \ldots \ldots$ & 0.05 & $0.01-0.1$ & $0.06-6.16$ & $0.08-0.84$ \\
\hline
\end{tabular}

NoTE.-The table contains parameter values in our standard model and its variants and their effect on the expected values of the $y$-parameter and peak opacity of the intergalactic dust to a source at $z=\infty$. The efficiency parameters $\eta_{y}$ and $\eta_{\tau}$ are defined in eqs. (11) and (13). Models with a predicted $y$-parameter in excess of the $C O B E$ upper limit, $y_{\mathrm{C}}<1.5 \times 10^{-5}$, are ruled out. The optical depth of intergalactic dust is significant and could affect observations with NGST. 


\section{OPTICAL DEPTH TO INFRARED SOURCES}

Next, we evaluate the optical depth to absorption and scattering by dust along the line of sight to a source at a redshift of $z_{\mathrm{s}}>3$,

$$
\tau_{\text {dust }}(v)=\frac{c}{H_{0}} \int_{3}^{z_{\mathrm{s}}} \frac{d z}{(1+z)^{5 / 2}} \alpha_{v(1+z)}(z) .
$$

We ignore contributions to the integral from $z<3$, since (1) the optical depth in homogeneously distributed dust at redshifts of $z_{\mathrm{s}}<3$ would be small, and (2) the spatial distribution of the attenuating dust at these redshifts is uncertain. Dust produced in massive galaxies with virial temperatures above $10^{5} \mathrm{~K}$ is unlikely to survive expulsion into the IGM through hot winds. Therefore, the additional dust produced at low redshifts is likely to be confined inside galaxies.

The absorption coefficient, $\alpha_{v(1+z)}(z)$, is given in equations (4) and (6). Since we are now interested in dust obscuration along a specific line of sight, both absorption and scattering must be included, and so we omit the last term in equation (6). The uncertainties discussed in $\S 2$ concerning the formation and survival of dust, as well as the fraction of the baryons converted into stars, affect the optical depth through the parameter, $\eta_{\tau}$,

$$
\eta_{\tau} \equiv f_{\text {surv }} f_{\text {flux }}\left(\frac{f_{\text {dust }}}{0.001}\right)\left(\frac{f_{\text {star }}}{0.13}\right)
$$

so that $\tau_{\text {dust }}$ obtains the value predicted by our standard model times, $\eta_{\tau}$.

Figure 2 shows the resulting optical depth in our standard model as a function of observed wavelength, $\lambda$, for three different source redshifts. The optical depth peaks at $\lambda \sim 1 \mu \mathrm{m}$, where it reaches a value of $\tau_{\text {dust }}=0.4$. This high value could suppress the Ly $\alpha$ emission peak in the spectra of early star clusters (see Charlot \& Fall 1991); attempts to detect this emission line from galaxies at $z \sim 2-4$ proved difficult in the past (Thompson, Djorgovski, \& Trauger 1995; Lowenthal et al. 1997). Since the total amount of dust is the same in all models with the same IMF, all other variations in the peak optical depths given in Table 1 arise from differences in the production history of dust. In particular, the optical depth is high when stars form early (e.g., as a result of a high $\sigma_{8 h^{-1}}$ or the lack of $\mathrm{H}_{2}$ destruction) and low when stars form late (low $n$ ). We note that the optical depths we derive are below the values that could cause a measurable reddening in the spectra of quasars at $z \lesssim 5$ (Rowan-Robinson 1995).

To illustrate the potential effect of dust on future observations with NGST, we show the composite spectrum of two star clusters, located at $z_{\mathrm{s}}=10$ and 20 and containing $10^{7}$ $M_{\odot}$ and $2.4 \times 10^{7} M_{\odot}$ in stars, respectively (Fig. 2, bottom panel). The stars are assumed to be distributed on the main sequence according to the Scalo IMF. In both cases the top curve shows the original unprocessed spectrum, and the bottom curve shows the spectrum obscured by the intervening dust in the standard model (with $\eta_{y}=1$ ). These results imply that the performance of NGST near its planned detection threshold of $1 \mathrm{nJy}$ in the wavelength range $1-3.5 \mu \mathrm{m}$ could be affected by intergalactic dust.

Another important conclusion that can be drawn from Figure 2 is that despite the obscuration by dust, the Lyman limit break could easily be identified in the emission spec-
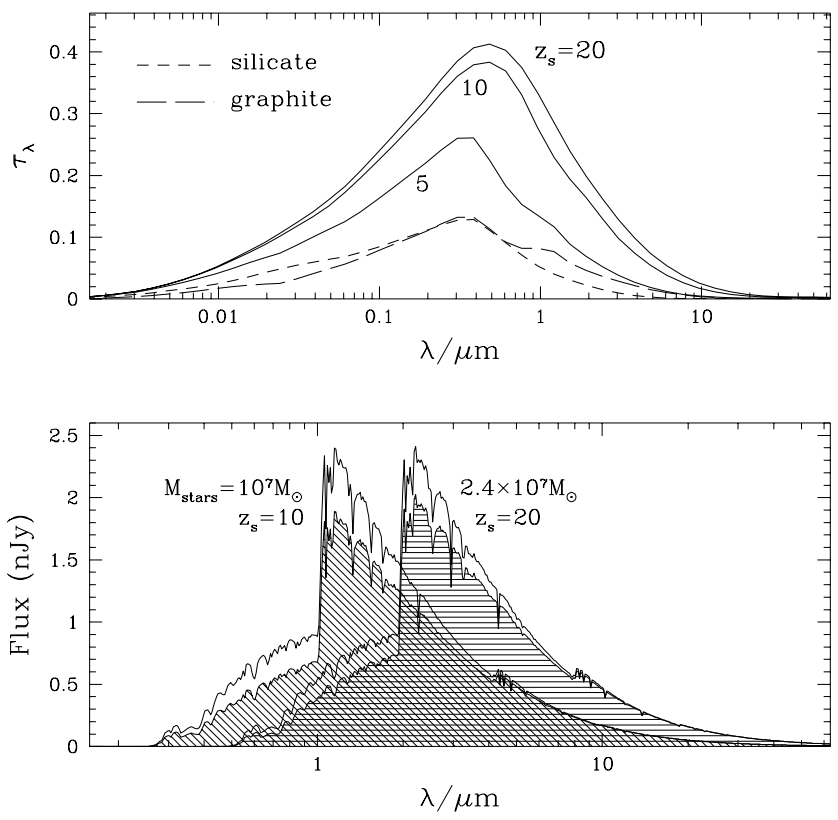

FIG. 2.-Top: Optical depth in our standard model to absorption and scattering by dust at $z \geq 3$, as a function of observed wavelength. The source is located at redshifts of $z_{s}=5,10$, or 20 . The short- and longdashed lines show the contributions from silicate and graphite dust grains for the $z=5$ case. Bottom: The expected flux with (lower curve) and without (upper curve) intervening dust, emitted from star clusters at $z_{s}=10$ and 20 , containing $10^{7} M_{\odot}$ and $2.4 \times 10^{7} M_{\odot}$ in stars. The stars are assumed to be distributed on the main sequence according to a Scalo IMF. The break in the spectra at the redshifted Lyman limit wavelength includes only the absorption by neutral hydrogen in the stellar atmospheres. Absorption by additional neutral hydrogen in or along the line of sight to the star cluster will modify the observed spectrum below the Ly $\alpha$ wavelength.

trum of star clusters at high redshifts. Additional absorption by neutral hydrogen in the IGM would only make the break appear sharper. For NGST observations between 1 and $3.5 \mu \mathrm{m}$, the detection of this break can identify source redshifts in the range $z_{\mathrm{s}}=10-35$. The feasibility of this $U V$ dropout technique was recently demonstrated through the successful photometric identification of galaxy redshifts in the interval $2.5 \lesssim z_{\mathrm{s}} \lesssim 3.5$ (Steidel et al. 1996; Madau et al. 1996).

\section{SUMMARY}

We have quantified the imprint of intergalactic dust from the first stars on the $y$-parameter of the CMB spectrum (in the $C O B E$ wavelength range), $y_{\mathrm{C}}$, and on the opacity to absorption and scattering of radiation from sources at high redshifts, $\tau_{\text {dust }}\left(z_{\mathrm{s}}\right)$. We normalized the star formation efficiency so as to get the observed $\sim 1 \%$ solar $\mathrm{C} / \mathrm{H}$ ratio in Ly $\alpha$ absorbers at $z=3$. With this normalization, we find $y_{\mathrm{C}} \sim 10^{-5}$ and $\tau_{\text {dust }}(\infty) \sim 1$ for plausible choices of the cosmological parameters and stellar properties at high redshifts (see Table 1). Uncertainties due to the mixing efficiency of metals with the IGM, the stellar IMF, the destruction of dust, the spatial correlations between dust and stellar sources, or the cosmological parameters could change these estimates by an order of magnitude. If early quasars exist, their additional UV radiation would enhance this spectral distortion.

The current $C O B E$ limit, $y_{\mathrm{C}}<1.5 \times 10^{-5}$, already places interesting constraints on the dust formation history of the 
universe at $z \gtrsim 10$. Complementary microwave anisotropy limits on the scale of $\sim 1^{\prime \prime}$ would constrain the degree of patchiness in the dust distribution. But most important, the future detection of source reddening at $z_{\mathrm{s}} \gtrsim 10$ by NGST, would be instrumental in establishing the properties of the first stars in the universe. Despite the significant dust opacity, Figure 2 shows that infrared photometry by NGST could identify the redshift of sources at $10 \lesssim z_{\mathrm{s}} \lesssim 35$, based on their pronounced Lyman limit break at observed wavelengths of $1-3.5 \mu \mathrm{m}$.

We thank Bruce Draine, Eli Dwek, Myungshin Im, Martin Rees, and Dimitar Sasselov for useful discussions. This work was supported in part by the NASA ATP grant NAG5-3085 and the Harvard Milton fund.
Adams, F. C., Freese, K., Levin, J., \& McDowell, J. 1989, ApJ, 344, 24

Bond, J. R., Carr, B. J., \& Hogan, C. J. 1991, ApJ, 367, 420

Charlot, S., \& Fall, M. S. 1991, ApJ, 378, 471

Cheney, J. E., \& Rowan-Robinson, M. 1981, MNRAS, 195, 831

Cowie, L. 1996, in Proc. 37th Herstmonceux Conf., HST and the High Redshift Universe, ed. N. R. Tanvir, A. Aragón-Salamanca, \& J. V. Wall (Singapore: World Scientific), in press

Draine, B. T., \& Lee, H. M. 1984, ApJ, 285, 89

Dwek, E. 1988, ApJ, 329, 814 .1997, in ASP Conf. Proc., Science with the Next Generation Space Telescope (San Francisco: ASP), in press

Eisenstein, D. J., \& Loeb, A. 1995, ApJ, 443, 11

Fixsen, D. J., et al. 1996, ApJ, 473, 576

Haiman, Z., \& Loeb, A. 1997, ApJ, 483, 21 (HL97)

Haiman, Z., Rees, M. J., \& Loeb, A. 1996, ApJ, 476, 458

Haiman, Z., Thoul, A., \& Loeb, A. 1996, ApJ, 464, 523

Hellsten, U., Dave, R., Hernquist, L., Weinberg, D. H., \& Katz, N. 1997, preprint, astro-ph/9701043

Kurucz, R. 1993, CD-ROM No. 13, ATLAS9 Stellar Atmosphere Programs

Lowenthal, J. D., et al. 1997, ApJ, 481, 673

Lu, L., Sargent, W. L. M., Barlow, T. A., Churchill, C. W., \& Vogt, S. S. 1996, ApJS, 107, 475

Lucy, L. B., Danziger, I. J., Gouiffes, C., \& Bouchet, P. 1991, in Supernovae, ed. S. E. Woosley (New York: Springer), 82

\section{REFERENCES}

Madau, P., Ferguson, H. C., Dickinson, M., Giavalisco, M., Steidel, C. C., \& Fruchter, A. 1996, MNRAS, 283, 1388

Mather, J., \& Stockman, P. 1996, STSci Newsletter, 13(2), 15

Mathis, J. S. 1990, ARA\&A, 28, 37

Miralda-Escudé, J., \& Rees, M. J. 1997, ApJ, 478, L57

Ostriker, J. P., \& Gnedin, N. 1996, ApJ, 472, 630

Ostriker, J. P., \& Heisler, J. 1984, ApJ, 278, 1

Ostriker, J. P., Vogeley, M. S., \& York, D. G. 1990, ApJ, 364, 405

Rauch, M., Haehnelt, M. G., \& Steinmetz, M. 1997, ApJ, 481, 601

Rowan-Robinson, M. 1995, MNRAS, 272, 737

Scalo, J. M. 1986, Fundam. Cosmic Phys., 11, 1

Schaller, G., Schaerer, D., Meynet, G., \& Maeder, A. 1992, A\&AS, 96, 269

Songaila, A., \& Cowie, L. L. 1996, AJ, 112, 335

Steidel, C. C., Giavalisco, M., Dickinson, M., \& Adelberger, K. L. 1996, AJ, 112,352

Thompson, D., Djorgovski, S., \& Trauger, J. 1995, AJ, 110, 963

Tytler, D. et al. 1995, in ESO Astrophysics Symposia, QSO Absorption Lines, ed. G. Meylan (Heidelberg: Springer), 289

Weaver, T. A., \& Woosley, S. E. 1980, Ann. NY Acad. Sci., 336, 335

Wright, E. L. 1981, ApJ, 250, 1 1990, ApJ, 353, 411

Wright, E. L., et al. 1994, ApJ, 420, 450 